\title{
Regularization for a Riesz-Feller space fractional backward diffusion problem with a time-dependent coefficient
}

- Dinh Nguyen Duy Hai

University of Science, VNU-HCM

Ho Chi Minh City University of Transport

(Received on $5^{\text {th }}$ December 2016, accepted on $28^{\text {th }}$ November 2017)

\begin{abstract}
In the present paper, we consider a backward problem for a space-fractional diffusion equation (SFDE) with a time-dependent coefficient. Such the problem is obtained from the classical diffusion equation by replacing the second-order spatial derivative with the Riesz-Feller derivative of order estimate, time-dependent coefficient

\section{INTRODUCTION}

The fractional differential equations appear more and more frequently in physical, chemical, biology and engineering applications. Nowadays, fractional diffusion equation plays important roles in modeling anomalous diffusion and subdiffusion systems [2], description of fractional random walk, unification of diffusion [3], and wave propagation phenomenon [4]. It is well known that the SFDE is obtained from the classical diffusion equation in which the second-order space derivative is replaced with a space-fractional partial derivative.
\end{abstract}

Key words: space-fractional backward diffusion problem, Ill-posed problem, Regularization, error

Let $\vartheta:[0, T] \rightarrow \mathbb{R}$ is a continuous function on $[0, T]$ satisfying $\vartheta(t)>0$. In this paper, we consider a $\alpha \in(0,2]$. This problem is ill-posed, i.e., the solution (if it exists) does not depend continuously on the data. Therefore, we propose one new regularization solution to solve it. Then, the convergence estimate is obtained under a priori bound assumptions for exact solution. backward problem for the following nonlinear SFDE with a time-dependent coefficient

$$
\left\{\begin{array}{l}
u_{t}(x, t)=\vartheta(t){ }_{x} D_{\theta}^{\alpha}+F(x, t, u(x, t)),(x, t) \in \mathbb{R} \times(0, T), \\
\left.u(x, t)\right|_{x \rightarrow \pm \infty}=0, t \in(0, T), \\
u(x, \mathrm{~T})=G(x), x \in \mathbb{R},
\end{array}\right.
$$

where the fractional spatial derivative ${ }_{x} D_{\theta}^{\alpha}$ is the Riesz-Feller fractional derivative of order $\alpha(0<\alpha \leq 2) \quad$ and skewness $\theta(|\theta| \leq \min \{\alpha, 2-\alpha\}, \theta \neq \pm 1)$ defined in [5], as follows:

\section{Trang 172}




$$
\left\{\begin{array}{l}
{ }_{x} D_{\theta}^{\alpha} f(x)=\frac{\Gamma(1+\alpha)}{\pi}\left\{\sin \frac{(\alpha+\theta) \pi}{2} \int_{0}^{\infty} \frac{f(x+s)-f(x)}{s^{1+\alpha}} d s+\sin \frac{(\alpha-\theta) \pi}{2} \int_{0}^{\infty} \frac{f(x-s)-f(x)}{s^{1+\alpha}} d s\right\}, 0<\alpha<2, \\
{ }_{x} D_{0}^{\alpha} f(x)=\frac{d^{2} f(x)}{d x^{2}}, \alpha=2 .
\end{array}\right.
$$

Here, we wish to determine the temperature $u(x, t)$ from temperature measurements $G^{\mathcal{E}}(x)$. Since the measurements usually contain an error, we now could assume that the measured data function $G^{\varepsilon}(x)$ satisfies $\left\|G-G^{\varepsilon}\right\|_{L^{2}(\mathbb{R})} \leq \varepsilon$, where the constant $\varepsilon>0$ represents the noise level. Moreover, assume there hold the following a priori bound

We assume that $F$ satisfies the Lipschitz condition

$$
\|u(\cdot, 0)\|_{L^{2}(\mathbb{R})} \leq E, E>\varepsilon .
$$

$$
\left\|F\left(x, t, z_{1}\right)-F\left(x, t, z_{2}\right)\right\|_{L^{2}(\mathbb{R})} \leq K_{F}\left\|z_{1}-z_{2}\right\|_{L^{2}(\mathbb{R})}
$$

for some constant $K_{F}$ independent of $x, t, z_{1}, z_{2}$ with

$$
K_{F} \in\left[0, \frac{1}{T}\right) \text {. }
$$

In case of the source function $F=0$ and $\vartheta(t)=1$, Problem (1) has been proposed by some authors. Zheng and Wei [7] used two methods, the spectral regularization and modified equation methods, to solve this problem. In [6], they developed an optimal modified method to solve this problem by an a priori and an a posteriori strategy. In 2014, Zhao et al [8] applied a simplified Tikhonov regularization method to deal with this problem. After then, a new regularization method of iteration type for solving this problem has been introduced by Cheng et al [1]. Although we have many works on the linear homogeneous case of the backward problem, the nonlinear case of the problem is quite scarce. For the nonlinear problem, the solution $u$ is complicated and defined by an integral equation such that the right hand side depends on $u$. This leads to studying nonlinear problem is very difficult, so in this paper we develop a new appropriate technique.
The remainder of this paper is organized as follows. In Section 2, we propose the regularizing scheme for Problem (1). Then, in Section 3, we show that the regularizing scheme of Problem (1) is wellposed. Finally, the convergence estimate is given in Section 4.

\section{REGULARIZATION FOR PROBLEM (1)}

Let $\hat{G}(\omega)$ denote the Fourier transform of the integrable function $G(x)$, which defined by

$$
\hat{G}(\omega):=\frac{1}{\sqrt{2 \pi}} \int_{-\infty}^{+\infty} \exp (-i x \omega) G(x) d x, \quad i=\sqrt{-1} .
$$

In terms of the Fourier transform, we have the following properties for the Riesz-Feller spacefractional derivative [5]

$$
{ }_{x} D_{\theta}^{\alpha}(G)(\omega)=-\psi_{\alpha}^{\theta}(\omega) \hat{G}(\omega),
$$

where

$$
\psi_{\alpha}^{\theta}(\omega)=|\omega|^{\alpha}\left[\cos \left(\frac{\theta \pi}{2}\right)+i \operatorname{sign}(\omega) \sin \left(\frac{\pi \theta}{2}\right)\right] .
$$

\section{Trang 173}


We define the function $k(t)$ by $k(t)=\int_{0}^{t} \frac{1}{\vartheta(s)} d s$.

By taking a Fourier transform to Problem (1), we transform Problem (1) into the following differential equation

$$
\left\{\begin{array}{l}
u_{t}(\omega, t)=-\vartheta(t) \psi_{\alpha}^{\theta}(\omega) \hat{u}(\omega, t)+\hat{F}(\omega, t, u(\omega, \mathrm{t})), \\
\hat{u}(\omega, \mathrm{T})=\hat{G}(\omega) .
\end{array}\right.
$$

The solution to equation (6) is given by

$$
\hat{u}(\omega, t)=\exp \left(\psi_{\alpha}^{\theta}(\omega)(k(T)-k(t))\right)\left[\hat{G}(\omega)-\int_{t}^{T} \exp \left(\psi_{\alpha}^{\theta}(\omega)(k(s)-k(T))\right) \hat{F}(\omega, s, u(\omega, s)) d s\right] .
$$

From (7), applying the inverse Fourier transform, we get

$$
u(x, t)=\frac{1}{\sqrt{2 \pi}} \int_{-\infty}^{+\infty} \exp \left(\psi_{\alpha}^{\theta}(\omega)(k(T)-k(t))\right)\left[\hat{G}(\omega)-\int_{t}^{T} \exp \left(\psi_{\alpha}^{\theta}(\omega)(k(s)-k(T))\right) \hat{F}(\omega, s, u(\omega, s)) d s\right] \exp (i x \omega) d \omega .
$$

From which when $|\omega|$ becomes large, the terms therefore recovering the scalar (temperature, pollution) $\left|\exp \left(\psi_{\alpha}^{o}(\omega)(k(T)-k(t))\right)\right|$ increases rather quickly: $u(x, t)$ from the measured data $G^{\varepsilon}(x)$ is severely illsmall errors in high-frequency components can blow up and completely destroy the solution for $0<t<T$, posed. In this note, we regularize Problem (1) by the problem

$$
\begin{aligned}
& U_{\beta}^{\varepsilon}(\omega, t)=\frac{\exp \left(\psi_{\alpha}^{\theta}(\omega)(k(T)-k(t))\right)}{1+\beta \exp \left(|\omega|^{\alpha} \cos \left(\frac{\theta \pi}{2}\right) k(T)\right)} G^{s}(\omega)-\int_{t}^{T} \frac{\exp \left(\psi_{\alpha}^{\theta}(\omega)(k(s)-k(t))\right)}{1+\beta \exp \left(|\omega|^{\alpha} \cos \left(\frac{\theta \pi}{2}\right) k(T)\right)} \hat{F}\left(\omega, s, U_{\beta}^{\varepsilon}(\omega, s)\right) d s \\
& +\int_{0}^{t} \frac{\beta \exp \left(|\omega|^{\alpha} \cos \left(\frac{\theta \pi}{2}\right) k(T)\right)}{1+\beta \exp \left(|\omega|^{\alpha} \cos \left(\frac{\theta \pi}{2}\right) k(T)\right)} \exp \left((k(s)-k(t)) \psi_{\alpha}^{\theta}(\omega)\right) \hat{F}\left(\omega, s, U_{\beta}^{s}(\omega, s)\right) d s,
\end{aligned}
$$

where $\beta$ is regularization parameter.

\section{THE WELL POSEDNESS OF PROBLEM (9)}

First, we consider the following Lemma which is used in the proof of the main results.

Lemma 1. Let $t, s \in[0, T]$.

1) If $s \geq t$, then we have

\section{Trang 174}


2) If $s \leq t$, then we have

$$
\begin{aligned}
& a)\left|\frac{\exp \left(\psi_{\alpha}^{\theta}(\omega)(k(s)-k(t))\right)}{1+\beta \exp \left(|\omega|^{\alpha} \cos \left(\frac{\pi \theta}{2}\right) k(T)\right)}\right| \leq \beta^{\frac{k(t)-k(s)}{k(T)}} . \\
& b)\left|\frac{\exp \left(\psi_{\alpha}^{\theta}(\omega)(k(T)-k(t))\right)}{1+\beta \exp \left(|\omega|^{\alpha} \cos \left(\frac{\pi \theta}{2}\right) k(T)\right)}\right| \leq \beta^{\frac{k(t)-k(T)}{k(T)}} .
\end{aligned}
$$

Proof. First, we prove (a). In fact, we have

$$
\text { c) }\left|\frac{\beta \exp \left(\psi_{\alpha}^{\theta}(\omega)(k(s)-k(t)+k(T))\right)}{1+\beta \exp \left(|\omega|^{\alpha} \cos \left(\frac{\theta \pi}{2}\right) k(T)\right)}\right| \leq \beta^{\frac{k(t)-k(s)}{k(T)}} .
$$

$$
\begin{aligned}
& \left|\frac{\exp \left(\psi_{\alpha}^{\theta}(\omega)(k(s)-k(t))\right)}{1+\beta \exp \left(|\omega|^{\alpha} \cos \left(\frac{\pi \theta}{2}\right) k(T)\right)}\right|=\frac{\exp \left(|\omega|^{\alpha} \cos \left(\frac{\pi \theta}{2}\right)(k(s)-k(t)-k(T))\right)}{\beta+\exp \left(-|\omega|^{\alpha} \cos \left(\frac{\pi \theta}{2}\right) k(T)\right)} \\
& =\frac{\exp \left(|\omega|^{\alpha} \cos \left(\frac{\pi \theta}{2}\right)(k(s)-k(t)-k(T))\right)}{\left[\beta+\exp \left(-|\omega|^{\alpha} \cos \left(\frac{\pi \theta}{2}\right) k(T)\right)\right]^{\frac{k(s)-k(t)}{k(T)}}\left[\beta+\exp \left(-|\omega|^{\alpha} \cos \left(\frac{\pi \theta}{2}\right) k(T)\right)\right]^{\frac{k(T)-k(s)+k(t)}{k(T)}}} \\
& \leq \frac{1}{\left[\beta+\exp \left(-|\omega|^{\alpha} \cos \left(\frac{\pi \theta}{2}\right) k(T)\right)\right]^{\frac{k(s)-k(t)}{k(T)}} \leq \beta^{\frac{k(t)-k(s)}{k(T)}} .}
\end{aligned}
$$

As an immediate consequence of (a), making the change $s=T$, we have (b).

Next, we prove (c). In fact from (b), we obtain

it follows that

$$
\left|\frac{\exp \left(\psi_{\alpha}^{\theta}(\omega)(k(T)-(k(t)-k(s)))\right)}{1+\beta \exp \left(|\omega|^{\alpha} \cos \left(\frac{\pi \theta}{2}\right) k(T)\right)}\right| \leq \beta^{\frac{k(t)-k(s)-k(T)}{k(T)}}
$$

$$
\left|\frac{\beta \exp \left(\psi_{\alpha}^{\theta}(\omega)(k(s)-k(t)+k(T))\right)}{1+\beta \exp \left(|\omega|^{\alpha} \cos \left(\frac{\pi \theta}{2}\right) k(T)\right)}\right| \leq \beta^{\frac{k(t)-k(s)}{k(T)}} .
$$

This completes the proof.

We are now in a position to prove the following theorem. 
Theorem 1. Suppose $m \in\left(0, \frac{1}{K_{F}^{2} T^{2}}-1\right)$. Let $G \in L^{2}(\mathbb{R})$ and $F$ satisfies (3) then Problem (9) is well-posed.

Proof. We divide it into two steps.

Step1. The existence and the uniqueness of a solution of Problem (9).

Let us define the norm on $C\left([0 ; T] ; L^{2}(\mathbb{R})\right)$ as follows

$$
\mid h\left\|_{0}=\sup _{0 \leq t \leq T} \beta^{\frac{-k(t)}{k(T)}}\right\| h(t) \|_{L^{2}(\mathbb{R})} \text {, for all } h \in C\left([0 ; T] ; L^{2}(\mathbb{R})\right) .
$$

It is easily be seen that I $\|_{0}$ is a norm of $C\left([0 ; T] ; L^{2}(\mathbb{R})\right)$.

For $v \in C\left([0 ; T] ; L^{2}(\mathbb{R})\right)$, we consider the following function

$$
\begin{aligned}
& A(v)(x, t)=\frac{1}{\sqrt{2 \pi}} B(x, t)-\frac{1}{\sqrt{2 \pi}} \int_{-\infty}^{+\infty} \int_{t}^{T} \frac{\exp \left(\psi_{\alpha}^{\theta}(\omega)(k(s)-k(t))\right)}{1+\beta \exp \left(|\omega|^{\alpha} \cos \left(\frac{\pi \theta}{2}\right) k(T)\right)} \hat{F}(\omega, s, v) \exp (i \omega x) d s d \omega \\
& +\frac{1}{\sqrt{2 \pi}} \int_{-\infty}^{+\infty} \int_{0}^{t} \frac{\beta \exp \left(|\omega|^{\alpha} \cos \left(\frac{\pi \theta}{2}\right) k(T)\right)}{1+\beta \exp \left(|\omega|^{\alpha} \cos \left(\frac{\pi \theta}{2}\right) k(T)\right)} \exp \left((k(s)-k(t)) \psi_{\alpha}^{\theta}(\omega)\right) \hat{F}(\omega, s, v) \exp (i \omega x) d s d \omega,
\end{aligned}
$$

where

$$
B(x, t)=\int_{-\infty}^{+\infty} \frac{\exp \left(\psi_{\alpha}^{\theta}(\omega)(k(T)-k(t))\right)}{1+\beta \exp \left(|\omega|^{\alpha} \cos \left(\frac{\pi \theta}{2}\right) k(T)\right)} G^{\varepsilon}(\omega) \exp (i \omega x) d \omega .
$$

We claim that, for every $v_{1}, v_{2} \in C\left([0 ; T] ; L^{2}(\mathbb{R})\right)$

$$
\text { | } A\left(v_{1}\right)-A\left(v_{2}\right)\left\|{ }_{0} \leq K_{F} T\right\| v_{1}-v_{2} \|_{0} .
$$

First, by Lemma 1 and (3), we have two following estimates for all $t \in[0, T]$

$$
J_{1}=\int_{-\infty}^{+\infty}\left(\int_{t}^{T} \frac{\exp \left(\psi_{\alpha}^{\theta}(\omega)(k(s)-k(t))\right)}{1+\beta \exp \left(|\omega|^{\alpha} \cos \left(\frac{\pi \theta}{2}\right) k(T)\right)}\left(\hat{F}\left(\omega, s, v_{1}\right)-\hat{F}\left(\omega, s, v_{2}\right)\right) d s\right)^{2} d \omega
$$

\section{Trang 176}




$$
\begin{aligned}
& \leq(T-t) \int_{-\infty}^{+\infty} \int_{t}^{T}\left|\frac{\exp \left(\psi_{\alpha}^{\theta}(\omega)(k(s)-k(t))\right)}{1+\beta \exp \left(|\omega|^{\alpha} \cos \left(\frac{\pi \theta}{2}\right) k(T)\right)}\right| \hat{F}\left(\omega, s, v_{1}\right)-\left.\hat{F}\left(\omega, s, v_{2}\right)\right|^{2} d s d \omega \\
& \leq(T-t) \int_{-\infty}^{+\infty} \int_{t}^{T} \beta^{\frac{2(k(t)-k(s))}{k(T)}}\left|\hat{F}\left(\omega, s, v_{1}\right)-\hat{F}\left(\omega, s, v_{2}\right)\right|^{2} d s d \omega \leq \beta^{\frac{2 k(t)}{k(T)}} K_{F}^{2}(T-t) \int_{t}^{T} \beta^{\frac{-2 k(s)}{k(T)}}\left\|v_{1}(\cdot, s)-v_{2}(\cdot, s)\right\|_{L^{2}(\mathbb{R})}^{2} d s \\
& \leq \beta^{\frac{2 k(t)}{k(T)}} K_{F}^{2}(T-t)^{2} \sup _{0 \leq s \leq T} \beta^{\frac{-2 k(s)}{k(T)}}\left\|v_{1}(\cdot, s)-v_{2}(\cdot, s)\right\|_{L^{2}(\mathbb{R})}^{2} \leq \beta^{\frac{2 k(t)}{k(T)}} K_{F}^{2}(T-t)^{2}\left\|v_{1}-v_{2}\right\|_{0}^{2}
\end{aligned}
$$

and

$$
\begin{aligned}
& J_{2}=\int_{-\infty}^{+\infty}\left(\int_{0}^{t} \frac{\beta \exp \left(|\omega|^{\alpha} \cos \left(\frac{\pi \theta}{2}\right) k(T)\right)}{1+\beta \exp \left(|\omega|^{\alpha} \cos \left(\frac{\pi \theta}{2}\right) k(T)\right)} \exp \left((k(s)-k(t)) \psi_{\alpha}^{\theta}(\omega)\right)\left(\hat{F}\left(\omega, s, v_{1}\right)-\hat{F}\left(\omega, s, v_{2}\right)\right) d s\right)^{2} d \omega \\
& \leq t \int_{-\infty}^{+\infty} \int_{0}^{t}\left|\frac{\beta \exp \left(|\omega|^{\alpha} \cos \left(\frac{\pi \theta}{2}\right) k(T)\right)}{1+\beta \exp \left(|\omega|^{\alpha} \cos \left(\frac{\pi \theta}{2}\right) k(T)\right)} \exp \left((k(s)-k(t)) \psi_{\alpha}^{\theta}(\omega)\right)\right| \hat{F}\left(\omega, s, v_{1}\right)-\left.\hat{F}\left(\omega, s, v_{2}\right)\right|^{2} d s d \omega \\
& \leq t \int_{-\infty}^{+\infty} \int_{0}^{+} \beta^{\frac{2(k(t)-k(s))}{k(T)}}\left|\hat{F}\left(\omega, s, v_{1}\right)-\hat{F}\left(\omega, s, v_{2}\right)\right|^{2} d s d \omega \leq \beta^{\frac{2 k(t)}{k(T)}} K_{F}^{2} t \int_{0}^{t} \beta^{\frac{-2 k(s)}{k(T)}}\left\|v_{1}(\cdot, s)-v_{2}(\cdot, s)\right\|_{L^{2}(\mathbb{R})}^{2} d s \\
& \leq \beta^{\frac{2 k(t)}{k(T)}} K_{F}^{2} t^{2} \sup _{0 \leq s \leq T} \beta^{\frac{-2 k(s)}{k(T)}}\left\|v_{1}(., s)-v_{2}(., s)\right\|_{L^{2}(\mathbb{R})}^{2} \leq \beta^{\frac{2 k(t)}{k(T)}} K_{F}^{2} t^{2}\left\|v_{1}-v_{2}\right\|_{0}^{2} .
\end{aligned}
$$

For $0<t<T$, using the inequality $(a+b)^{2} \leq(1+m) a^{2}+\left(1+\frac{1}{m}\right) b^{2}$ for all real numbers $a$ and $b$ and $m>0$, we obtain

$$
\left\|A\left(v_{1}\right)(\cdot, t)-A\left(v_{2}\right)(\cdot, t)\right\|_{L^{(}(\mathbb{R})}^{2} \leq(1+m) \beta^{\frac{2 k(t)}{k(T)}} K_{F}^{2} t^{2}\left\|v_{1}-v_{2}\right\|_{0}^{2}+\left(1+\frac{1}{m}\right) \beta^{\frac{2 k(t)}{k(T)}} K_{F}^{2}(T-t)^{2}\left\|v_{1}-v_{2}\right\|_{0}^{2} .
$$

By choosing $m=\frac{T-t}{t}$, we have

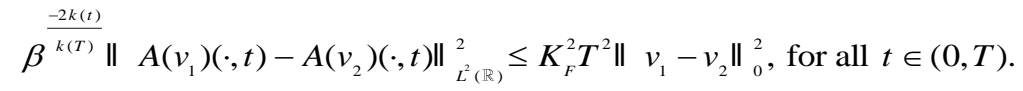

On the other hand, letting $t=0$ in (11), we have

$$
\text { | } A\left(v_{1}\right)(\cdot, 0)-A\left(v_{2}\right)(\cdot, 0)\left\|_{L^{2}(\mathbb{R})}^{2} \leq K_{F}^{2} T^{2}\right\| v_{1}-v_{2} \|_{0}^{2} .
$$

By letting $t=T$ in (12), we have

$$
\beta^{-2}\left\|A\left(v_{1}\right)(\cdot, T)-A\left(v_{2}\right)(\cdot, T)\right\|_{L^{2}(\mathbb{R})}^{2} \leq K_{F}^{2} T^{2}\left\|v_{1}-v_{2}\right\|_{0}^{2} .
$$

Combining (13), (14) and (15), we obtain 


$$
\beta^{\frac{-2 k(t)}{k(T)} \|} A\left(v_{1}\right)(\cdot, t)-A\left(v_{2}\right)(\cdot, t)\left\|_{L^{2}(\mathbb{R})}^{2} \leq K_{F}^{2} T^{2}\right\| v_{1}-v_{2} \|_{0}^{2} \text {, for all } t \in[0, T]
$$

which leads to (10). Since $K_{F} T<1, A$ is a contraction. It follows that the equation $A(v)=v$ has a unique solution $U_{\beta}^{\varepsilon} \in C\left([0 ; T] ; L^{2}(\mathbb{R})\right)$.

Step 2. The solution of Problem (9) continuously depends on the data.

Let $V_{\beta}^{\varepsilon}, W_{\beta}^{\varepsilon}$ be two solutions of Problem (9) corresponding to the final values $G_{V}$ and $G_{W}$. By straightforward computation, we write

$$
\begin{aligned}
\left|W_{\beta}^{\varepsilon}(\omega, t)-V_{\beta}^{\varepsilon}(\omega, t)\right| \leq\left|\frac{\exp \left(\psi_{\alpha}^{\theta}(\omega)(k(T)-k(t))\right)}{1+\beta \exp \left(|\omega|^{\alpha} \cos \left(\frac{\pi \theta}{2}\right) k(T)\right)}\left(G_{W}(\omega)-G_{V}(\omega)\right)\right| \\
+\left|\int_{t}^{T} \frac{\exp \left(\psi_{\alpha}^{\theta}(\omega)(k(s)-k(t))\right)}{1+\beta \exp \left(|\omega|^{\alpha} \cos \left(\frac{\pi \theta}{2}\right) k(T)\right)}\left[\hat{F}\left(\omega, s, V_{\beta}^{\varepsilon}(\omega, s)\right)-\hat{F}\left(\omega, s, W_{\beta}^{\varepsilon}(\omega, s)\right)\right] d s\right| \\
+\left|\int_{0}^{t} \frac{\beta \exp \left(|\omega|^{\alpha} \cos \left(\frac{\pi \theta}{2}\right) k(T)\right)}{1+\beta \exp \left(|\omega|^{\alpha} \cos \left(\frac{\pi \theta}{2}\right) k(T)\right)} \exp \left((k(s)-k(t)) \psi_{\alpha}^{\theta}(\omega)\right)\left[\hat{F}\left(\omega, s, W_{\beta}^{\varepsilon}(\omega, s)\right)-\hat{F}\left(\omega, s, V_{\beta}^{\varepsilon}(\omega, s)\right)\right] d s\right| .
\end{aligned}
$$

Now applying Lemma 1, we get

$$
\begin{aligned}
& \left|W_{\beta}^{\varepsilon}(\omega, t)-V_{\beta}^{\varepsilon}(\omega, t)\right| \leq \beta^{\frac{k(t)-k(T)}{k(T)}}\left|G_{W}(\omega)-G_{V}(\omega)\right|+\int_{t}^{T} \beta^{\frac{k(t)-k(s)}{k(T)}}\left|\hat{F}\left(\omega, s, V_{\beta}^{\varepsilon}(\omega, s)\right)-\hat{F}\left(\omega, s, W_{\beta}^{\varepsilon}(\omega, s)\right)\right| d s \\
& +\int_{0}^{t} \beta^{\frac{k(t)-k(s)}{k(T)}}\left|\hat{F}\left(\omega, s, V_{\beta}^{\varepsilon}(\omega, s)\right)-\hat{F}\left(\omega, s, W_{\beta}^{\varepsilon}(\omega, s)\right)\right| d s \\
& \leq \beta
\end{aligned}
$$

Since $m \in\left(0, \frac{1}{K_{F}^{2} T^{2}}-1\right)$, we have that $0<K_{F}<\frac{1}{T \sqrt{1+m}}$. From the inequality

$$
(a+b)^{2} \leq\left(1+\frac{1}{m}\right) a^{2}+(1+m) b^{2}
$$

for all real number $a, b$ and $m>0$, we get

$$
\left\|W_{\beta}^{\varepsilon}(\cdot, t)-V_{\beta}^{\varepsilon}(\cdot, t)\right\|_{L^{2}(\mathbb{R})}^{2}=\left\|W_{\beta}^{\varepsilon}(\cdot, t)-V_{\beta}^{\varepsilon}(\cdot, t)\right\|_{L^{2}(\mathbb{R})}^{2}
$$

\section{Trang 178}




$$
\begin{aligned}
& \leq\left(1+\frac{1}{m}\right) \beta^{\frac{2 k(t)-2 k(T)}{k(T)}}\left\|G_{W}-G_{V}\right\|_{L^{2}(\mathbb{R})}^{2}+(1+m) \int_{-\infty}^{+\infty}\left(\int_{0}^{T} \beta^{\frac{k(t)-k(s)}{k(T)}}\left|\hat{F}\left(\omega, s, V_{\beta}^{\varepsilon}(\omega, s)\right)-\hat{F}\left(\omega, s, W_{\beta}^{\varepsilon}(\omega, s)\right)\right| d s\right)^{2} d \omega \\
& \leq\left(1+\frac{1}{m}\right) \beta^{\frac{2 k(t)-2 k(T)}{k(T)}}\left\|G_{W}-G_{V}\right\|_{L^{2}(\mathbb{R})}^{2}+(1+m) K_{F}^{2} T \int_{0}^{T} \beta^{\frac{2 k(t)-2 k(s)}{k(T)}}\left\|W_{\beta}^{\varepsilon}(\cdot, s)-V_{\beta}^{\varepsilon}(\cdot, s)\right\|_{L^{2}(\mathbb{R})}^{2} d s .
\end{aligned}
$$

This leads to

$$
\beta^{\frac{-2 k(t)}{k(T)}}\left\|W_{\beta}^{\varepsilon}(\cdot, t)-V_{\beta}^{\varepsilon}(\cdot, t)\right\|_{L^{2}(\mathbb{R})}^{2} \leq\left(1+\frac{1}{m}\right) \beta^{-2}\left\|G_{W}-G_{V}\right\|_{L^{2}(\mathbb{R})}^{2}+(1+m) K_{F}^{2} T \int_{0}^{T} \beta^{\frac{-2 k(s)}{k(T)}}\left\|W_{\beta}^{\varepsilon}(\cdot, s)-V_{\beta}^{\varepsilon}(\cdot, s)\right\|_{L^{2}(\mathbb{R})}^{2} d s
$$

Set $Z(t)=\beta^{\frac{-2 k(t)}{k(T)}}\left\|W_{\beta}^{\varepsilon}(\cdot, t)-V_{\beta}^{\varepsilon}(\cdot, t)\right\|_{L^{2}(\mathbb{R})}^{2}, \forall t \in[0, T]$. Since $W_{\beta}^{\varepsilon}, V_{\beta}^{\varepsilon} \in C\left([0, T] ; L^{2}(\mathbb{R})\right)$, we see that the function $Z$ is continuous on $[0, T]$ and attains over there its maximum $M$ at some $t_{0} \in[0, T]$. Let $M=\max _{t \in[0, T]} Z(t)$. From (17), we obtain

$$
M \leq\left(1+\frac{1}{m}\right) \beta^{-2}\left\|G_{W}-G_{V}\right\|_{L^{2}(\mathbb{R})}^{2}+(1+m) K_{F}^{2} T^{2} M
$$

or equivalently

$$
\left[1-(1+m) K_{F}^{2} T^{2}\right] M \leq\left(1+\frac{1}{m}\right) \beta^{-2}\left\|G_{W}-G_{V}\right\|_{L^{2}(\mathbb{R})}^{2} .
$$

This implies that for all $t \in[0, T]$

$$
\beta^{\frac{-2 k(t)}{k(T)}}\left\|W_{\beta}^{\varepsilon}(\cdot, t)-V_{\beta}^{\varepsilon}(\cdot, t)\right\|_{L^{2}(\mathbb{R})}^{2} \leq M \leq \frac{\left(1+\frac{1}{m}\right) \beta^{-2}\left\|G_{W}-G_{V}\right\|_{L^{2}(\mathbb{R})}^{2}}{1-(1+m) K_{F}^{2} T^{2}} .
$$

Thus, we obtain

$$
\left\|W_{\beta}^{\varepsilon}(\cdot, t)-V_{\beta}^{\varepsilon}(\cdot, t)\right\|_{L^{2}(\mathbb{R})} \leq \sqrt{\frac{1+\frac{1}{m}}{1-(1+m) K_{F}^{2} T^{2}}} \beta^{\frac{k(t)-k(T)}{k(T)}}\left\|G_{W}-G_{V}\right\|_{L^{2}(\mathbb{R})}, \forall t \in[0, T] .
$$

This completes the proof of Step 2 and also the proof of the theorem.

\section{CONVERGENCE ESTIMATE}

Now we are ready to state the main result

Theorem 2. Let $m \in\left(0, \frac{1}{K_{F}^{2} T^{2}}-1\right)$. Suppose that Problem (1) has a unique solution $u \in C\left([0, T] ; L^{2}(\mathbb{R})\right)$ satisfying $\|u(\cdot, 0)\|_{L^{2}(\mathbb{R})} \leq E$ with $E>\varepsilon$ and the regularization parameter $\beta=\frac{\varepsilon}{E}$ then we have the estimate 


$$
\left\|U_{\beta}^{\varepsilon}(\cdot, t)-u(\cdot, t)\right\|_{L^{2}(\mathbb{R})} \leq 2 \sqrt{\frac{1+\frac{1}{m}}{1-(1+m) K_{F}^{2} T^{2}}} \varepsilon^{\frac{k(t)}{k(T)}} E^{1-\frac{k(t)}{k(T)}} .
$$

Proof. Assuming that $u_{\beta}^{\varepsilon}$ is a solution of Problem (9) corresponding to the final values $G$, we shall estimate $\left\|u(\cdot, t)-u_{\beta}^{\varepsilon}(\cdot, t)\right\|_{L^{2}(\mathbb{R})}$. First we have

$$
\begin{aligned}
\hat{u}(\omega, t) & =\exp \left(\psi_{\alpha}^{\theta}(\omega)(k(T)-k(t))\right)\left(\hat{G}(\omega)-\int_{t}^{T} \exp \left(\psi_{\alpha}^{\theta}(\omega)(k(s)-k(T))\right) \hat{F}(\omega, s, u(\omega, s)) d s\right) \\
= & \frac{\exp \left(\psi_{\alpha}^{\theta}(\omega)(k(T)-k(t))\right)}{1+\beta \exp \left(|\omega|^{\alpha} \cos \left(\frac{\pi \theta}{2}\right) k(T)\right)}\left(\hat{G}(\omega)-\int_{t}^{T} \exp \left(\psi_{\alpha}^{\theta}(\omega)(k(s)-k(T))\right) \hat{F}(\omega, s, u(\omega, s)) d s\right) \\
& +\frac{\beta \exp \left(\psi_{\alpha}^{\theta}(\omega)(k(T)-k(t))\right) \exp \left(|\omega|^{\alpha} \cos \left(\frac{\pi \theta}{2}\right) k(T)\right)}{1+\beta \exp \left(|\omega|^{\alpha} \cos \left(\frac{\pi \theta}{2}\right) k(T)\right)}\left(\hat{G}(\omega)-\int_{t}^{T} \exp \left(\psi_{\alpha}^{\theta}(\omega)(k(s)-k(T))\right) \hat{F}(\omega, s, u(\omega, s)) d s\right) .
\end{aligned}
$$

On the other hand, we get

$$
\hat{u}(\omega, T)=\hat{G}(\omega)=\exp \left(-k(T) \psi_{\alpha}^{\theta}(\omega)\right)\left(\hat{u}(\omega, 0)+\int_{0}^{T} \exp \left(k(s) \psi_{\alpha}^{\theta}(\omega)\right) \hat{F}(\omega, s, u(\omega, s)) d s\right) .
$$

This implies that

$$
\begin{aligned}
& \hat{G}(\omega)-\int_{t}^{T} \exp \left(\psi_{\alpha}^{\theta}(\omega)(k(s)-k(T))\right) \hat{F}(\omega, s, u(\omega, s)) d s \\
& =\exp \left(-k(T) \psi_{\alpha}^{\theta}(\omega)\right) \hat{u}(\omega, 0)+\int_{0}^{t} \exp \left((k(s)-k(T)) \psi_{\alpha}^{\theta}(\omega)\right) \hat{F}(\omega, s, u(\omega, s)) d s .
\end{aligned}
$$

Combining (19) and (20), we obtain

$$
\begin{gathered}
\hat{u}(\omega, t)=\frac{\exp \left(\psi_{\alpha}^{\theta}(\omega)(k(T)-k(t))\right)}{1+\beta \exp \left(|\omega|^{\alpha} \cos \left(\frac{\pi \theta}{2}\right) k(T)\right)}\left(\hat{G}(\omega)-\int_{t}^{T} \exp \left(\psi_{\alpha}^{\theta}(\omega)(k(s)-k(T))\right) \hat{F}(\omega, s, u(\omega, s)) d s\right) \\
+\frac{\beta \exp \left(-k(t) \psi_{\alpha}^{\theta}(\omega)\right) \exp \left(|\omega|^{\alpha} \cos \left(\frac{\pi \theta}{2}\right) k(T)\right)}{1+\beta \exp \left(|\omega|^{\alpha} \cos \left(\frac{\pi \theta}{2}\right) k(T)\right)} \hat{u}(\omega, 0) \\
+\frac{\beta \exp \left(|\omega|^{\alpha} \cos \left(\frac{\pi \theta}{2}\right) k(T)\right)}{1+\beta \exp \left(|\omega|^{\alpha} \cos \left(\frac{\pi \theta}{2}\right) k(T)\right)} \int_{0}^{t} \exp \left((k(s)-k(t)) \psi_{\alpha}^{\theta}(\omega)\right) \hat{F}(\omega, s, u(\omega, s)) d s .
\end{gathered}
$$

\section{Trang 180}


It follows from (9) and (21) that $\hat{u}(\omega, t)-u_{\beta}^{\varepsilon}(\omega, t)=B_{1}+B_{2}+B_{3}$,

where

$$
\begin{aligned}
& B_{1}=\int_{t}^{T} \frac{\exp \left(\psi_{\alpha}^{\theta}(\omega)(k(s)-k(t))\right)}{1+\beta \exp \left(|\omega|^{\alpha} \cos \left(\frac{\pi \theta}{2}\right) k(T)\right)}\left[\hat{F}\left(\omega, s, u_{\beta}^{\varepsilon}(\omega, s)\right)-\hat{F}(\omega, s, u(\omega, s))\right] d s, \\
& B_{2}=\frac{\beta \exp \left(-k(t) \psi_{\alpha}^{\theta}(\omega)\right) \exp \left(|\omega|^{\alpha} \cos \left(\frac{\pi \theta}{2}\right) k(T)\right)}{1+\beta \exp \left(|\omega|^{\alpha} \cos \left(\frac{\pi \theta}{2}\right) k(T)\right)} \hat{u}(\omega, 0), \\
& B_{3}=\frac{\beta \exp \left(|\omega|^{\alpha} \cos \left(\frac{\pi \theta}{2}\right) k(T)\right)}{1+\beta \exp \left(|\omega|^{\alpha} \cos \left(\frac{\pi \theta}{2}\right) k(T)\right)} \int_{0}^{t} \exp \left((k(s)-k(t)) \psi_{\alpha}^{\theta}(\omega)\right)\left[\hat{F}(\omega, s, u(\omega, s))-\hat{F}\left(\omega, s, u_{\beta}^{\varepsilon}(\omega, s)\right)\right] d s .
\end{aligned}
$$

This leads to

$$
\begin{aligned}
& \left|\hat{u}(\omega, t)-u_{\beta}^{\varepsilon}(\omega, t)\right| \leq\left|B_{1}\right|+\left|B_{2}\right|+\left|B_{3}\right| \\
& \leq \int_{t}^{T} \beta^{\frac{k(t)-k(s)}{k(T)}}\left|\hat{F}(\omega, s, u(\omega, s))-\hat{F}\left(\omega, s, u_{\beta}^{\varepsilon}(\omega, s)\right)\right| d s+\beta^{\frac{k(t)}{k(T)}}|\hat{u}(\cdot, 0)|+\int_{0}^{t} \beta^{\frac{k(t)-k(s)}{k(T)}}\left|\hat{F}(\omega, s, u(\omega, s))-\hat{F}\left(\omega, s, u_{\beta}^{\varepsilon}(\omega, s)\right)\right| d s \\
& \leq \beta^{\frac{k(t)}{k(T)}}|\hat{u}(\cdot, 0)|+\int_{0}^{T} \beta^{\frac{k(t)-k(s)}{k(T)}}\left|\hat{F}(\omega, s, u(\omega, s))-\hat{F}\left(\omega, s, u_{\beta}^{\varepsilon}(\omega, s)\right)\right| d s .
\end{aligned}
$$

Using this and (16), we conclude that

$$
\begin{aligned}
& \left\|u(\cdot, t)-u_{\beta}^{\varepsilon}(\cdot, t)\right\|_{L^{2}(\mathbb{R})}^{2}=\left\|\hat{u}(\cdot, t)-u_{\beta}^{\varepsilon}(\cdot, t)\right\|_{L^{2}(\mathbb{R})}^{2}
\end{aligned}
$$

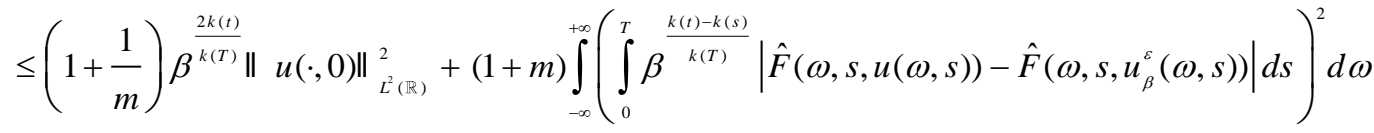

$$
\begin{aligned}
& \leq\left(1+\frac{1}{m}\right) \beta^{\frac{2 k(t)}{k(T)}}\|u(\cdot, 0)\|_{L^{2}(\mathbb{R})}^{2}+(1+m) K_{F}^{2} T \beta^{\frac{2 k(t)}{k(T)}} \int_{0}^{T} \beta^{\frac{-2 k(s)}{k(T)}}\left\|u(\cdot, s)-u_{\beta}^{\varepsilon}(\cdot, s)\right\|_{L^{2}(\mathbb{R})}^{2} d s,
\end{aligned}
$$

and thus

$$
\beta^{\frac{-2 k(t)}{k(T)}}\left\|u(\cdot, t)-u_{\beta}^{\varepsilon}(\cdot, t)\right\|_{L^{2}(\mathbb{R})}^{2} \leq\left(1+\frac{1}{m}\right)\|u(\cdot, 0)\|_{L^{2}(\mathbb{R})}^{2}+(1+m) K_{F}^{2} T \int_{0}^{T} \beta^{\frac{-2 k(s)}{k(T)}}\left\|u(\cdot, s)-u_{\beta}^{\varepsilon}(\cdot, s)\right\|_{L^{2}(\mathbb{R})}^{2} d s .
$$


Since $u, u_{\beta}^{\varepsilon} \in C\left([0, T] ; L^{2}(\mathbb{R})\right)$, the function $\left\|u(\cdot, t)-u_{\beta}^{\varepsilon}(\cdot, t)\right\|_{L^{2}(\mathbb{R})}$ is continuous on $[0, T]$. Therefore, there exists a positive $N=\max _{t \in[0, T]} \beta^{\frac{-2 k(t)}{k(T)}}\left\|u(\cdot, t)-u_{\beta}^{\varepsilon}(\cdot, t)\right\|_{L^{2}(\mathbb{R})}^{2}$. This implies that

$$
N \leq\left(1+\frac{1}{m}\right)\|u(\cdot, 0)\|_{L^{2}(\mathbb{R})}^{2}+(1+m) K_{F}^{2} T^{2} N,
$$

that is,

$$
\beta^{\frac{-2 k(t)}{k(T)}}\left\|u(\cdot, t)-u_{\beta}^{\varepsilon}(\cdot, t)\right\|_{L^{2}(\mathbb{R})}^{2} \leq N \leq \frac{\left(1+\frac{1}{m}\right)\|u(\cdot, 0)\|_{L^{2}(\mathbb{R})}^{2}}{1-(1+m) K_{F}^{2} T^{2}} .
$$

Hence, we obtain the error estimate

$$
\left\|u(\cdot, t)-u_{\beta}^{\varepsilon}(\cdot, t)\right\|_{L^{2}(\mathbb{R})} \leq E \sqrt{\frac{1+\frac{1}{m}}{1-(1+m) K_{F}^{2} T^{2}}} \beta^{\frac{k(t)}{k(T)}} .
$$

On the other hand, using estimate (18), we get

$$
\left\|u_{\beta}^{\varepsilon}(\cdot, t)-U_{\beta}^{\varepsilon}(\cdot, t)\right\|_{L^{2}(\mathbb{R})} \leq \sqrt{\frac{1+\frac{1}{m}}{1-(1+m) K_{F}^{2} T^{2}}} \beta^{\frac{k(t)-k(T)}{k(T)}}\left\|G^{\varepsilon}-G\right\|_{L^{2}(\mathbb{R})} \leq \sqrt{\frac{1+\frac{1}{m}}{1-(1+m) K_{F}^{2} T^{2}} \beta^{\frac{k(t)-k(T)}{k(T)}}} \varepsilon .
$$

From the triangle inequality and these estimates, we obtain

$$
\left\|U_{\beta}^{\varepsilon}(\cdot, t)-u(\cdot, t)\right\|_{L^{2}(\mathbb{R})} \leq\left\|U_{\beta}^{\varepsilon}(\cdot, t)-u_{\beta}^{\varepsilon}(\cdot, t)\right\|_{L^{2}(\mathbb{R})}+\left\|u_{\beta}^{\varepsilon}(\cdot, t)-u(\cdot, t)\right\|_{L^{2}(\mathbb{R})}
$$

$\leq \sqrt{\frac{1+\frac{1}{m}}{1-(1+m) K_{F}^{2} T^{2}}} \beta^{\frac{k(t)-k(T)}{k(T)}} \varepsilon+E \sqrt{\frac{1+\frac{1}{m}}{1-(1+m) K_{F}^{2} T^{2}}} \beta^{\frac{k(t)}{k(T)}}$.

With $\beta=\frac{\varepsilon}{E}$, then we have the estimate

$$
\left\|U_{\beta}^{\varepsilon}(\cdot, t)-u(\cdot, t)\right\|_{L^{2}(\mathbb{R})} \leq 2 \sqrt{\frac{1+\frac{1}{m}}{1-(1+m) K_{F}^{2} T^{2}}} \varepsilon^{\frac{k(t)}{k(T)}} E^{1-\frac{k(t)}{k(T)}} .
$$

This completes the proof.

Remark 1. If $\vartheta(t)=1$ and $F(x, t, u)=0$ then Problem (1) becomes a homogeneous problem. The error estimate in Theorem 2 is of order $\beta^{\frac{t}{T}}$. It is similar to the homogeneous case in $[1,6,8]$.

\section{CONCLUSION}

In this paper, we use the new regularization solution to slove a Riesz-Feller space-fractional backward diffusion problem with a time-dependent coefficient. The convergence result has been obtained under a priori bound assumptions for the exact solution and the suitable choices of the regularization parameter.

\section{Trang 182}




\section{Chỉnh hóa cho bài toán khuếch tán ngược cấp phân số không gian Riesz-Feller với hệ số phụ thuộc thời gian}

- Đinh Nguyễn Duy Hải

Trường Đại học Khoa học Tự nhiên, ĐHQG-HCM

Trường Đại học Giao thông Vận tải Tp Hồ Chí Minh

\section{TÓM TẮT}

Trong bài báo này, chúng tôi xét một bài toán nguợc cho phuơng trình khuếch tán cấp phân số không gian với hệ số phu thuộc thời gian. Bài toán này có được tù phuơng trình khuếch tán cổ điển bằng cách thay đạo hàm bậc hai biến không gian bằng đạo hàm Riesz-Feller với $\alpha \in(0,2]$. Đây là bài toán không

Tù khóa: bài toán khuếch tán ngược cấp phân số không gian, bài toán không chỉnh, chỉnh hóa, uớc luợng lỗi, hệ số phu thuộc thời gian

\section{TÀI LIỆU THAM KHẢO}

[1]. H. Cheng, C.L. Fu, G.H. Zheng, J. Gao, A regularization for a Riesz-Feller space-fractional backward diffusion problem, Inverse Probl. Sci. Eng., 22, 860-872 (2014).

[2]. O.P. Agrawal, Solution for a fractional diffusionwave equation defined in a bounded domain, Nonlinear Dynamics, 29, 145-155 (2002).

[3]. R. Metzler, J. Klafter, The random walk's guide to anomalous diffusion: a fractional dynamics approach, Physical Reports, 339, 1-77 (2000).

[4]. WR. Schneider, W. Wyss, Fractional diffusion and wave equations, Journal of Mathematical Physics, 30, 134- 144 (1989).

[5]. F. Mainardi, Y. Luchko, G. Pagnini, The fundamental solution of the space-time fractional chỉnh, nghĩa là nghiệm (nếu tồn tại) không phu thuộc liên tục vào dũ liệu. Vì vậy, chúng tôi đưa ra một nghiệm chỉnh hóa mói để giải bài toán này. Sau đó, uớc luợng hội tu thu được duoói một giả định bị chặn tiên nghiệm cho nghiệm chính xác. diffusion equation, Fract. Cacl. Appl. Anal., 4, 153192 (2001).

[6]. Z.Q. Zhang, T. Wei, An optimal regularization method for space-fractional backward diffusion problem, Math. Comput. Simulation, 92, 14-27 (2013).

[7]. G.H. Zheng, T. Wei, Two regularization methods for solving a Riesz-Feller space-fractional backward diffusion problem, Inverse Problems, 26, 115017 (2010).

[8]. J. Zhao, S. Liu, T. Liu, An inverse problem for space-fractional backward diffusion problem, Math. Methods Appl. Sci., 37, 1147- 1158 (2014). 